\title{
Variability and Evidence of Non-spherical Stellar Winds in A-Type Supergiants
}

\author{
Eva Verdugo $^{1}$, Antonio Talavera ${ }^{2}$, and Ana I. Gómez de Castro ${ }^{3}$ \\ 1 ISO Data Centre, VILSPA, P.O. Box 50727, 28080 Madrid, Spain \\ 2 LAEFF/INTA, VILSPA, P.O. Box 50727, 28080 Madrid, Spain \\ 3 Instituto de Astronomía y Geodesia (CSIC-UCM), Fac. cc. Matemáticas, \\ Universidad Complutense, Av. Complutense s/n, E-28040-Madrid, Spain
}

\begin{abstract}
The profiles of the $\mathrm{H} \alpha$ and uv lines and the observed variability suggest deviations from spherical symmetry for the envelope of A-type supergiants.
\end{abstract}

\section{Introduction}

During the last years increasing efforts have been dedicated to study the atmospheres of A-type supergiants. However, compared to the amount of work devoted to OB stars, studies of photospheric and wind properties of A-type supergiants are still scarce.

In this paper we describe several visible and ultraviolet spectral lines of a sample of 41 A supergiants. The spectral ranges observed include the Balmer lines ( $\mathrm{H} \alpha, \mathrm{H} \beta, \mathrm{H} \delta$ and $\mathrm{H} \gamma$ ), the $\mathrm{Ca}$ II $\mathrm{H}$ and $\mathrm{K}$ lines, the $\mathrm{Na}$ I D lines and the Mg II line at $4481 \AA$ and the uv spectra obtained with the IUE satellite. These observations represent the best source of data so far for a global view of their spectral variability.

We also present the first results of a quantitatively analysis of the $\mathrm{H} \alpha$ profile by means of a non-LTE model.

\section{Line profiles}

The most sensitive indicator of winds in A supergiants is the $\mathrm{H} \alpha$ line in the visible range and the $\mathrm{Mg}$ II (uv1) lines in the ultraviolet range. Our analysis of these lines shows that these stars can be divided into two groups: the less luminous stars (spectral type Ib) which show symmetric absorption profiles in all the visible lines studied, and only show wind effects in the uv lines of Mg II as a time evolving component. The second group contains the most luminous A supergiants (spectral type Ia and Iab). The $\mathrm{H} \alpha$ line in these stars presents asymmetric, P Cygni and/or double-peaked emission profiles. The uv lines (Mg II, C II, Si II, Al II and Fe II) are also asymmetric with no emission and a sharp blue edge or profiles composed of discret absorption components. These profiles are represented in Fig. 1.

The frontier between these two groups is $M_{v} \sim-6$. 


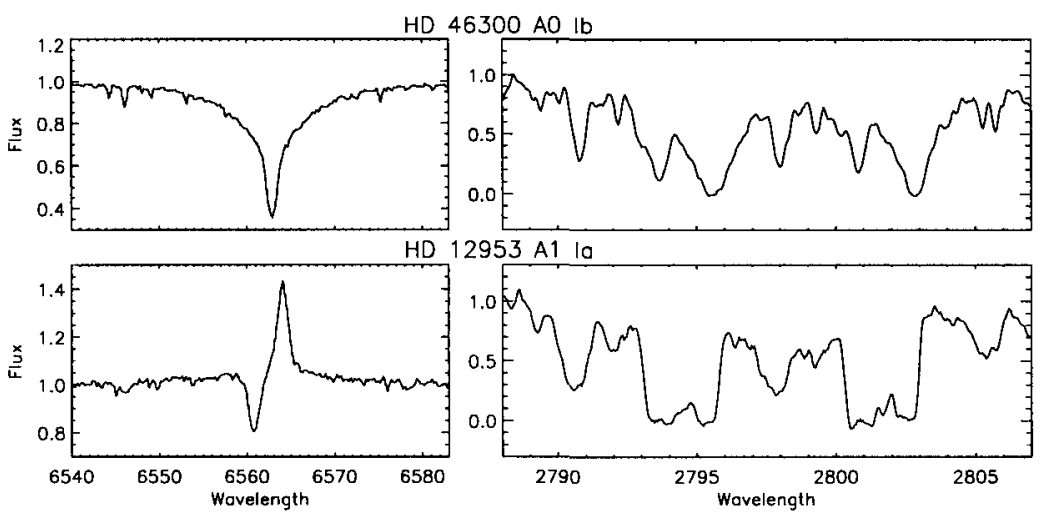

Fig. 1. $\mathrm{H} \alpha$ and Mg II (uv1) profiles for Group I (top) and Group II (bottom).

\section{Variability}

In the visible range we have only found significant variations in the $\mathrm{H} \alpha$ line. Stars with symmetric absorption $\mathrm{H} \alpha$ profiles do not show any variability. However we have observed variations in most of the stars showing emission profiles. The strongest variations are detected on time-scales of days and most of them are due to a variable double-peaked emission. The observed $\mathrm{V} / \mathrm{R}$ variability points out deviations from spherical symmetry (Fig. 2).

Contrary to what woud be expected we found that the less luminous stars present the most spectacular variations in the uv spectrum. We have observed the appearance and evolution of a DAC in the $\mathrm{Mg} I I$ lines (Fig. 2). However such component have not been detected in any other uv line.

The time-scales of variability are consistent with the rotational periods.

\begin{tabular}{lcccc}
\hline Object & $v_{\text {break }}[\mathrm{km} / \mathrm{s}]$ & $P_{\text {break }}[$ days $]$ & $v \sin i[\mathrm{~km} / \mathrm{s}]$ & $P_{\text {rot }} / \sin i[$ days] \\
\hline \hline HD 87737 & 202 & 10.5 & 20 & 106 \\
HD 12953 & 147 & 50 & 49 & 150 \\
HD 207260 & 175 & 26 & 44 & 102 \\
\hline
\end{tabular}

\section{Synthetic $\mathrm{H} \alpha$ profiles}

We have modelling $\mathrm{H} \alpha$ profiles for an expanding, spherically symmetric atmosphere. We have used the equivalent-two-level-atom approach (Mihalas and Kunasz, 1978) to solve the radiative transfer problem and the non-LTE equation of statistical equilibrium in the context of a multilevel hydrogen atom. The transfer equation is solved in the comoving frame of the flow.

We have found several models that match the observed emission strength (Fig. 3). However we have encountered difficulty to math the depth of the absorption feature. Even the most succesful profiles are deeper than the observed profiles suggesting that these models do not include all the physical 

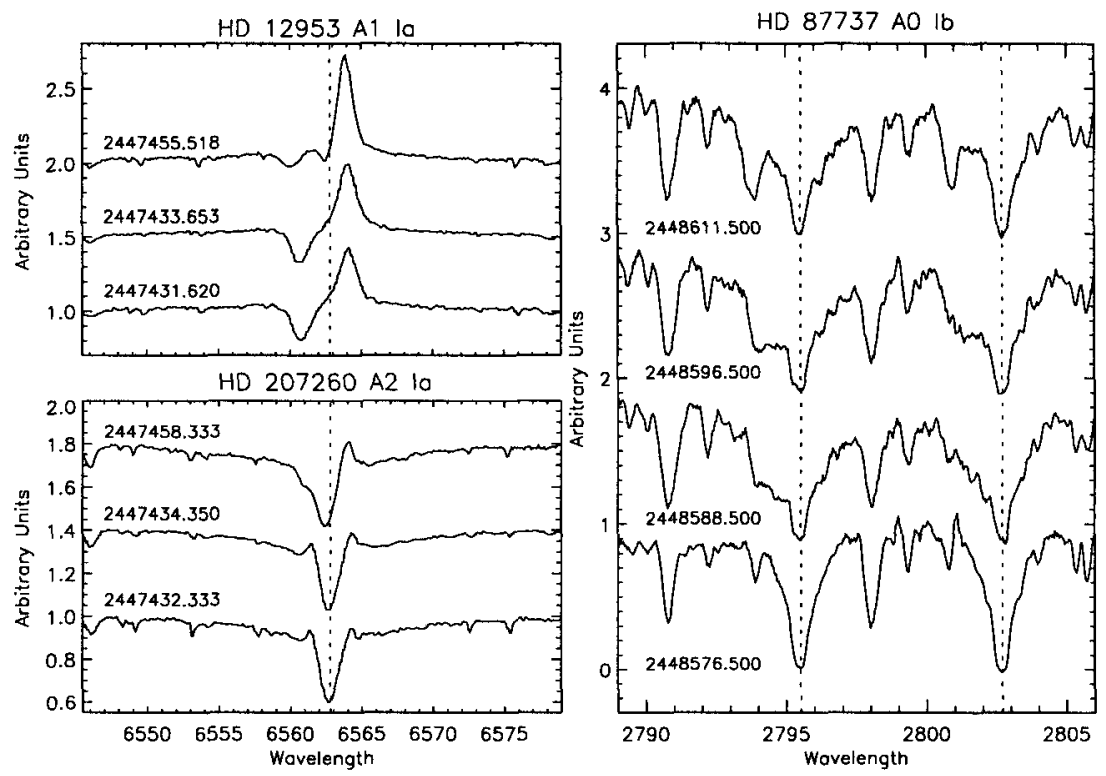

Fig. 2. Variability in the $\mathrm{H} \alpha$ and $\mathrm{Mg}$ II (uv1) profiles. The rest position of the lines are marked with a dashed line. Each spectrum is labeled with the Julian Date of the observation.

processes that operate in the envelope of A-type supergiants. Deviations from steady-state flow structure and non-spherical extension are possible.
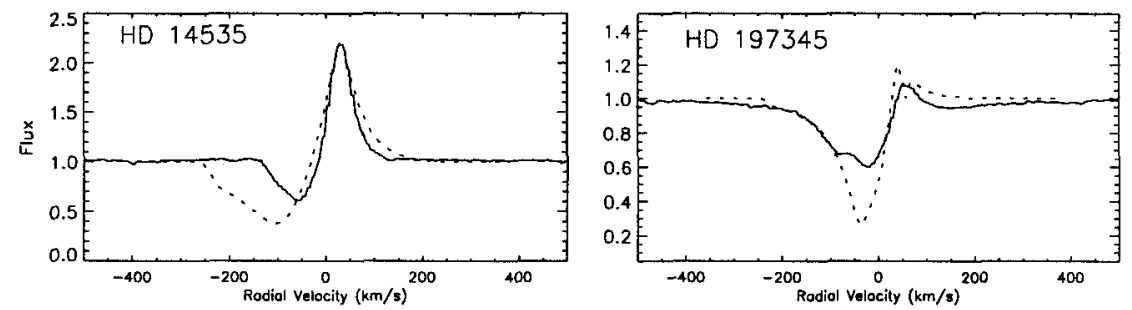

Fig. 3. Synthetic $\mathrm{H} \alpha$ (dashed line) compared with the observations (solid line).

\section{References}

Mihalas, D., Kunasz, P.B.: 1978, Astrophys. J., 219, 635 


\section{Discussion}

L. Kaper: What fraction of your sample of A supergiants shows doublepeaked $\mathrm{H} \alpha$ emission? How do you interpret this?

E. Verdugo: About ten stars of our sample display double-peaked $\mathrm{H} \alpha$ emission. Presently, I cannot explain this. I can only say that these kind of profiles are the main evidence for deviations from spherical symmetry for the envelopes of A supergiants. In fact, we cannot reproduce these profiles with the ETLA code, which assumes an expanding spherically, symmetric atmosphere.

J. Puls: In A super/hypergiants, the $\mathrm{H} \alpha$ profile should have a $\mathrm{P}$ Cygni morphology, for theoretical reasons. However, as you have shown, these spectra exhibit pure emission profiles at $\mathrm{H} \alpha$. Do you have an idea of a reason for the behaviour?

E. Verdugo: No, I have no explanation for this behaviour.

H. Lamers: Maybe clumping.

S. Shore: What is the frequency with which you find the absorption components that look sort of detached? Are they variable? (This could be an indication of clumping in the wind.)

E. Verdugo: I have only detected such a profile once, for two stars of my sample: HD 12953, which has a variable P Cygni profile; and HD 223960, which shows the strongest double-peaked emission profile with clear $V / R$ variability.

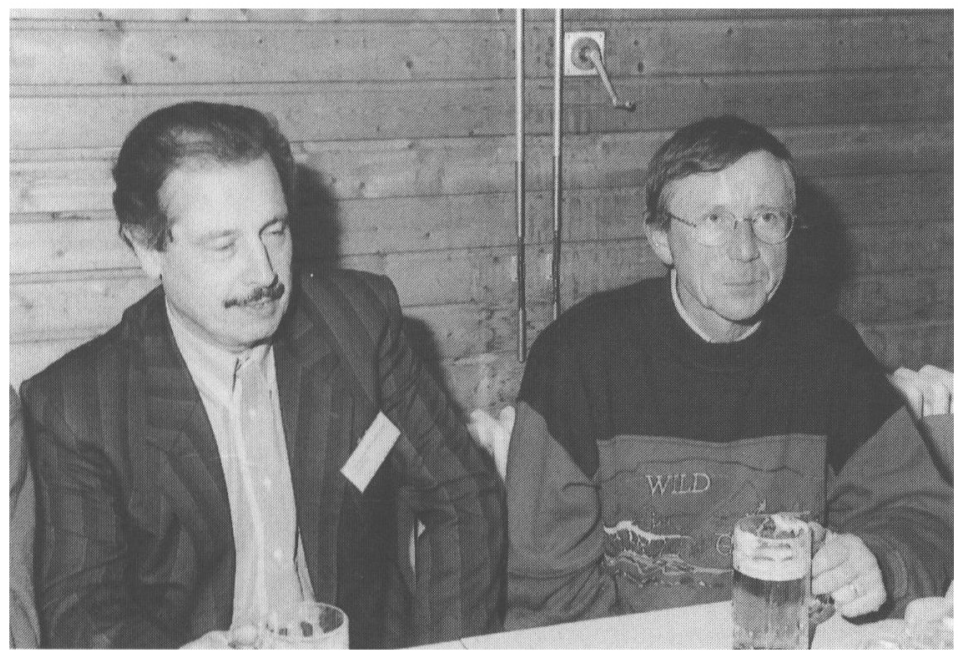

Jaques Breysacher and Christiaan Sterken 